\title{
Wi-Fi All-Channel Analyzer
}

\author{
Sergio Barrachina-Muñoz, Boris Bellalta, Edward Knightly
}

\section{Abstract}

In this paper, we present WACA, the first system to simultaneously measure the energy in all $24 \mathrm{Wi}-\mathrm{Fi}$ channels that allow channel bonding at $5 \mathrm{GHz}$ with microsecond scale granularity. With WACA, we perform a first-of-its-kind measurement campaign in areas including urban hotspots, residential neighborhoods, universities, and a sold-out stadium with 98,000 fans and 12,000 simultaneous Wi-Fi connections. The gathered dataset is a unique asset to find insights otherwise not possible in the context of multi-channel technologies like Wi-Fi. To show its potential, we compare the performance of contiguous and non-contiguous channel bonding using a trace-driven framework. We show that while non-contiguous outperforms contiguous channel bonding's throughput, occasionally bigger by a factor of 5 , their average throughputs are similar.

Keywords: channel bonding, measurement campaign, spectrum occupancy, IEEE 802.11ax, Camp Nou stadium

\section{Introduction}

Channel bonding is a key mechanism for increasing Wi-Fi data rates as the maximum data rate increases in proportion to the total channel bandwidth. In Wi-Fi, while the basic channel width remains $20 \mathrm{MHz}$, the maximum bonded channel width has increased from $40 \mathrm{MHz}$ in $802.11 \mathrm{n}$ [1] to $160 \mathrm{MHz}$ in 802.11ac/ax [2, 3], and $320 \mathrm{MHz}$ in 802.11be [4]. During this time, the standard has evolved to not only support wider bandwidths, but also to enable more sophisticated channel bonding policies: in $802.11 n$, only "static" channel bonding was allowed in which a fixed group of pre-configured channels must always be bonded. Today, the standard enables a far richer set of capabilities including dynamic selection of channel width as well as bonding both contiguous and non-contiguous channels.

In this paper, we make the following three contributions. First, we develop WACA as the first system to simultaneously measure all $24 \mathrm{Wi}-\mathrm{Fi}$ channels at $5 \mathrm{GHz}$ with a $10 \mu \mathrm{sec}$ sampling rate. WACA employs multiple synchronized WARP software defined radios (SDRs) and has 24 antennas and 24 radio frequency (RF) chains to match the number of Wi-Fi channels that allow channel bonding (from channel 36 to 161) in the IEEE 802.11ac/ax standards. Our approach contrasts with prior work that uses one or several RF chains, thereby encountering second scale channel switching delays. Thus, no prior methods capture all channels at the channel-access $\mu$ sec timescale.

Second, using WACA, we conduct extensive measurement campaigns covering two continents, dense urban areas, and places of interest such as university campuses, apartment buildings, shopping malls, and hotels. We also perform measurements in the Futbol Club Barcelona's Camp Nou, one of the largest sports stadiums in the world. The shortest campaign took 20 minutes and the longest covered more than 1 week, and the total number of samples exceeds $10^{11}$. In all cases, we record signal strength on all channels at $10 \mu \mathrm{sec}$ sample rate, from which we infer the epochs for which each channel is occupied 正 Notice that the traces compose an unprecedented dataset to find insights otherwise not possible. We highlight $\mathrm{Wi}-\mathrm{Fi}$ as the main research application of the dataset but others can also be taken into account, e.g., cognitive radios, coexistence between Wi-Fi and LTE, and coexistence between Wi-Fi and Internet of Things (IoT) technologies, to list a few examples.

Third, we introduce a trace-driven framework to evaluate the performance of channel bonding policies using the aforementioned high-resolution traces of channel activity. As the channel occupancies are highly dynamic, we group epochs according to their average utilization. While the stadium measurements have band occupancies as high as $99 \%$, even dense urban scenarios with many competing basic service sets (BSS's) yielded maximum occupancies near $45 \%$.

Finally, we use the trace-driven framework to compare the performance of contiguous vs. non-contiguous channel bonding and find that the increased flexibility of the latter can yield a throughput improvement of up to $5 \times$. Unfortunately, the scenarios for these gains occur quite rarely yielding modest average gains of less than $10 \%$, which may ultimately favor contiguous channel bonding, since it is simpler to implement.

Due to space constrains, we leave the study of key factors such as spectral correlation, bandwidth prevention, or standard compliant channel bonding policies as future work.

\footnotetext{
${ }^{1}$ The WACA dataset v1 can be found at https://www.upf .edu/web/ wnrg/wn-datasets.

${ }^{2}$ All of the source code of WACA is open, encouraging sharing of algorithms between contributors and providing the ability for people to improve on the work of others under the GNU General Public License v3.0. The repository can be found at https://github.com/ sergiobarra/WACA_WiFiAnalyzer.
} 


\section{Related work}

Prior work performed spectrum measurements for Wi-Fi traffic, e.g., [5, 6, 7, 8, 9, 10, 11, 12, 13, 14, 15]. Example objectives include creating interference maps [15], assessing interference behavior [16], surveying Wi-Fi usage [10, quantifying spectrum occupancy in outdoor testbeds [5, 6], designing efficient scanning methods [7, 11, modeling spectrum use [8, opportunistic spectrum access 9, 14, dynamic channel selection [17, and assessing real-world network behavior by examining data from thousands APs [12]. Unfortunately, no such prior work provided simultaneous measurement across the entire $5 \mathrm{GHz}$ band, which we require for our channel bonding study. While some papers do provide multi-channel measurements, e.g., [18, 14, 9, 5], they do so via sequential scanning, thus taking on the order of seconds to change from one channel to the next, orders of magnitude beyond the transmission time scale for channel bonding. Namely, WACA measures all channels simultaneously using SDRs having a sampling rate of $10 \mu$ sec. Moreover, prior work does not consider (for example) stadiums.

Throughput gains of channel bonding have been demonstrated previously in testbeds. In particular, IEEE 802.11n static channel bonding has been shown to be affected not only by link signal quality, but also by the power and rates of neighboring links [19]. Likewise, intelligent channel bonding management was shown to benefit from identifying the signal strength of neighboring links and interference patterns [20]. High bandwidths were shown to be vulnerable to increased thermal noise or the power per Hertz reduction [21, 22]. Nonetheless, existing experimental results have targeted only on one or few controlled links at most. In contrast, we develop WACA as a monitoring system in order to measure multiple BSS's in various operational settings, which allows tackling how a channel bonding BSS interacts with surrounding BSS's in a broad set of scenarios.

Simulation studies and analytical models have also been employed to study channel bonding, e.g., early simulation studies demonstrated throughput gains of channel bonding compared to single-channel transmission [23, 24]. Likewise, analytical models have been proposed to study channel bonding, especially through Markov chains [25, 26]. Analytical models for unsaturated traffic have been also proposed [27, 28]. As well, different channel bonding policies were introduced and modeled for spatially distributed scenarios 29. However, such work does not have an experimental validation as presented here. In this regard, WACA datasets can be used for validation of the aforementioned methods under real-world channel occupancies in future work.

\section{Wi-Fi All-Channel Analyzer}

\subsection{Overview}

Our objective is to simultaneously capture activity on all Wi-Fi channels, i.e., all 24 basic (non aggregated) $20 \mathrm{MHz}$ channels in the $5 \mathrm{GHz}$ band that permit channel bonding. In principle, this could be achieved with an off-the-shelf spectrum analyzer. However, most spectrum analyzers are not capable of dealing with the required bandwidth of this objective, i.e., they cannot simultaneously measure the entire Wi-Fi 5-GHz band: $645 \mathrm{MHz}$ ranging from channel 36 to 161 (i.e., from 5170 to $5815 \mathrm{MHz}$ ). Moreover, wide-band spectrum analyzers that can cover this bandwidth lack resolution to analyze basic channels within the band.

Likewise, one could envision a system comprising 24 off-the shelf Wi-Fi cards as sniffers, one per basic channel. Unfortunately, such a system would be quite unwieldy and would introduce a challenge of ensuring synchronicity among wireless cards: restricting the delay between channel measurements to the order of nano/microseconds is unfeasible due to the hardware interrupt latency and jitter from the different peripherals 30, 31].

Thus, we design WACA to simultaneously measure power (and I/Q signals if required) on all $5-\mathrm{GHz}$ basic channels. Key benefits of WACA include the simplicity of experimental procedures (from deployment to post-processing), a dedicated RF chain per channel (covering the whole band and easing hardware failure detection), and the ease of adaptation/configuration empowered by the WARPLAb framework 32 .

\subsection{Building Blocks}

The key building blocks of WACA are $i$ ) six WARP v3 programmable wireless SDRs 33, ii) six FMC-RF-2X245 dualradio FMC daughterboards ${ }^{3}$ iii) $245-\mathrm{GHz}$ antennas (one per RF chain), and $i v$ ) one Ethernet switch to enable communication between the WARPLab host (e.g., PC) and the WARP boards. The preeminent building block is WARP, a scalable and extensible programmable wireless platform to prototype advanced wireless networks. The FMC-RF2X245 module dual-radio FPGA Mezzanine Card (FMC) daughterboard extends the capability of WARP v3 boards from 2 to $4 \mathrm{RF}$ chains. Therefore, by combining 2 stacks of 3 WARP boards each with their corresponding FMC-RF2X245 daughterboards, we realize $24 \mathrm{RF}$ chains (with one $5-\mathrm{GHz}$ antenna each) enabling us to assign a single RF chain per basic channel. Finally, the Ethernet switch enables the communication from the WARP nodes to the WARPLab host. Figure 1a shows the assignment of the RF chains to each basic channel allowed for bonding and Figure $1 \mathrm{~b}$ depicts the physical realization of WACA.

\subsection{Measurement Methodology}

An iterative procedure is followed for collecting power samples. Namely, in each iteration, WACA first simultaneously measures the power at each basic channel during $T_{\mathrm{m}}$ and then takes $T_{\text {proc }}$ to process and forward the measurements to the

\footnotetext{
${ }^{3}$ FMC-RF-2X245 datasheet: https://mangocomm.com/products/ modules/fmc-rf-2x245, retrieved January 30, 2020.
} 


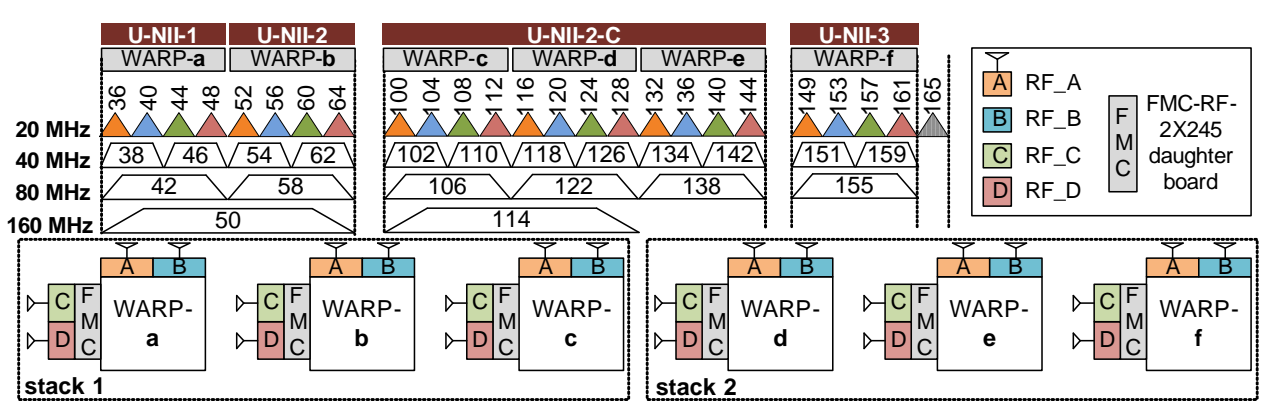

(a) IEEE $802.11 \mathrm{ac} / \mathrm{ax}$ channelization at the $5-\mathrm{GHz}$ and assignation per RF.

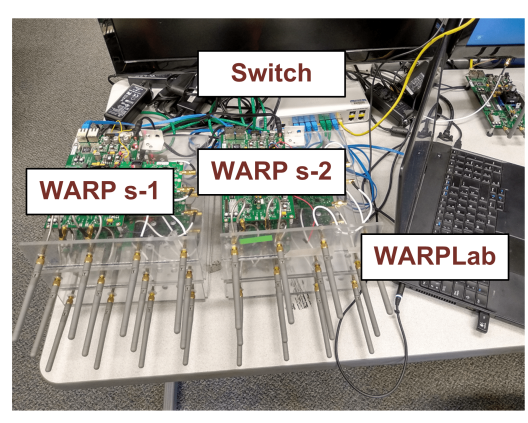

(b) Deployment schematic.

Figure 1: The WACA all WiFi channels spectrum analyzer.

\begin{tabular}{cc}
\hline Parameter & Value \\
\hline Active RFs (channels 36 to 161), $\mathcal{R}$ & $\{1,2, \ldots, 24\}$ \\
Iteration's measurement duration, $T_{\mathrm{m}}$ & $1 \mathrm{~s}$ \\
Iteration's processing duration, $T_{\text {proc }}$ & $\sim 9 \mathrm{~s}$ \\
Duration of a complete iteration, $T_{\mathrm{it}}$ & $\sim 10 \mathrm{~s}$ \\
$\quad$ Original no. of power samples & $10^{7}$ \\
$\quad$ per channel per second, $n_{\mathrm{s}}^{*}$ & \\
Downsampled no. of power samples & $10^{5}$ \\
$\quad$ per channel per second, $n_{\mathrm{s}}$ & $10 \mu \mathrm{s}$ \\
\hline Time between consecutive samples, $T_{\mathrm{s}}$ & $125-59500$ \\
\hline Number of iterations, $N_{\mathrm{it}}$ &
\end{tabular}

Table 1: WARPLab setup.

WARPLab host. Both tasks are sequentially performed until the end of the measurement campaign. Table 1 shows the main WARPLab parameters used for measurements. Notice that all parameters are fixed except $N_{\mathrm{it}}$, used for determining the duration of the measurement campaign.

WARP boards install the MAX2829 transceiver, which has a fixed 10 Msps received signal strength sampling rate. Accordingly, since the measurement duration in an iteration is $T_{\mathrm{m}}=1$ second, the number of consecutive samples captured per basic channel per iteration is $n_{\mathrm{s}}^{*}=10^{7}$. Then, in each iteration, we store $|\mathcal{R}| \times n_{\mathrm{s}}^{*}=24 \times 10^{7}$ samples. Nonetheless, to decrease the amount of required memory, we downsample the gathered samples in each iteration by a $100 \times$ factor, thus reducing the data size per iteration from 60 MB to $600 \mathrm{~KB}$. Essentially, while the transceiver measures 1 power sample every $100 \mathrm{~ns}$ by default, we keep just 1 sample every $T_{s}=10 \mathrm{\mu s}$. Notice that the resulting time scale is also suitable given Wi-Fi timings. Indeed, the short interframe space (SIFS) is the smallest interframe space and takes $16 \mu \mathrm{s}\left(>T_{s}\right)$.

As for the duration of processing and forwarding (period in which no data is collected), $T_{\text {proc }}$ entails a significant yet unavoidable delay overhead with respect to the total duration of an iteration $T_{\mathrm{it}}=T_{\mathrm{m}}+T_{\mathrm{proc}}$. Specifically, for the host PC used in all the scenarios in the dataset (Intel Core i5-4300U CPU @ 1.9 GHz x 4 and 7.7 GiB memory) and the parameters listed in Table 1. $T_{\text {proc }} \approx 9 \mathrm{~s}$ for $T_{\mathrm{m}}=1 \mathrm{~s}$. Once initiated, WACA operates by itself, and no human intervention is required.

\subsection{Validation}

Before deploying WACA for the measurement campaigns, we perform an extensive set of in-lab controlled experiments for validation, comprising over $6 \times 10^{8}$ power samples collected in WACA cross validated with controlled and known transmissions from commercial APs. We conduct the validation of all the boards by first measuring the power perceived against distance and transmission bandwidth. Then, we explore the spectrum behavior when setting up different off-the-shelf channel bonding configurations. The measurements were gathered in an empty and large event room (about $300 \mathrm{~m}^{2}$ ). Figure 2 shows the complete deployment. In particular, we deploy WACA including the PC hosting the WARPLab framework (lower part of the figure), 3 PCs receiving iperf traffic (right part of the figure), and 3 PCs sending iperf traffic with the corresponding 3 APs enabling the iperf connections (left part of the figure). The AP models we use are Asus RT-AC87U (AS), TP-Link AC1750 (TP), and Linksys WRT 3200 ACM (LS). Remarkably, all of these APs are only capable of performing static channel bonding.

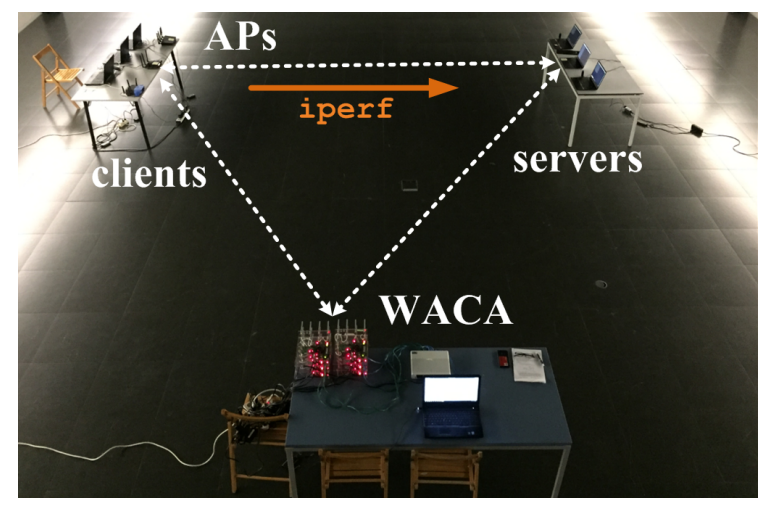

Figure 2: Validation testbed at the main events room of Universitat Pompeu Fabra's Communication campus. 


\subsubsection{RSSI vs. central frequency}

Ideally, we would like each of the $24 \mathrm{RFs}$ to perceive exactly the same RSSI value when receiving the same signal modulated at a central frequency $f$. Besides, we would also like each of the RFs to perceive exactly the same RSSI value at every central frequency $f$ when the same signal is modulated at each $f$. In other words, the RSSI vs. $f$ should be the same flat curve for all the RFs. In order to validate such premises, we use a WARP node acting as a transmitter and sequentially connect the transmitter RF (which keeps generating the same $20-\mathrm{MHz}$ signal) to each of the $24 \mathrm{RFs}$ of WACA for every channel from 36 to 161 via an RF-SMA connector. As shown in Figure 3, a similar RSSI is perceived at every RF. Besides, a similar flat frequency response is achieved for every RF.

\subsubsection{RSSI vs. distance}

We measure the RSSI in $\mathrm{dBm}^{4}$ at each basic channel at different distances $d$ from the AP to WACA ranging from 1.2 to 22.8 meters. We took $10^{5}$ samples per point. For redundancy, we use $4 \mathrm{RFs}$ to measure the same channel $p=1245^{5}$ As expected, the RSSI tends to decrease with the distance due to the path loss effect as shown in Figure 3b For the sake of representation, we also plot the simplified path loss (SPL) of the mean power received at each point: $\operatorname{RSSI}(d)=\operatorname{RSSI}\left(d_{0}\right)-\alpha 10 \log _{10}(d)$, with $d=1 \mathrm{~m}$. Despite the experiments were conducted in a large, empty room without furniture, we can see the multipath effects in the sparse RSSI values perceived by each RF due to the reflection of the walls. Such effect is anticipated since the minimum separation between RFs is $6 \mathrm{~cm}$.

\subsubsection{RSSI vs. bandwidth}

This experiment aims at measuring the RSSI perceived at a fixed distance $(3.6 \mathrm{~m})$ in four basic channels at the same time for 20,40 , and $80 \mathrm{MHz}$ transmissions. The general procedure followed in the experiment can be summarized in 4 steps: $i$ ) the AS AP is set up with bandwidth $b \in\{20,40,80\} \mathrm{MHz}$ and primary channel $p=124$; ii) an iperf client is placed close to the AP and associates to it; iii) an iperf server is located at distance $d=3.6 \mathrm{~m}$ from the AP and also associates to it; finally, $i v$ ) the iperf communication is triggered and WACA captures the resulting RSSI at channels 116, 120, 124, and 128 , thus covering $80 \mathrm{MHz}$. Notice that it is enough to use just one WARP board with the corresponding daughterboard to cover the 4 basic channels of interest. Theoretically, when doubling the bandwidth, the transmission power gets reduced by a half $(3 \mathrm{~dB})$. We corroborate this fact by looking at the similar reduction factor in the power detected per $\mathrm{Hz}$ in Figure $3 \mathrm{c}$

\footnotetext{
${ }^{4}$ We follow the MAX2829 transceiver data sheet to convert from 10-bit RSSI values to $\mathrm{dBm}$ values as done in [34].

${ }^{5}$ We selected this channel since negligible activity was detected.
}

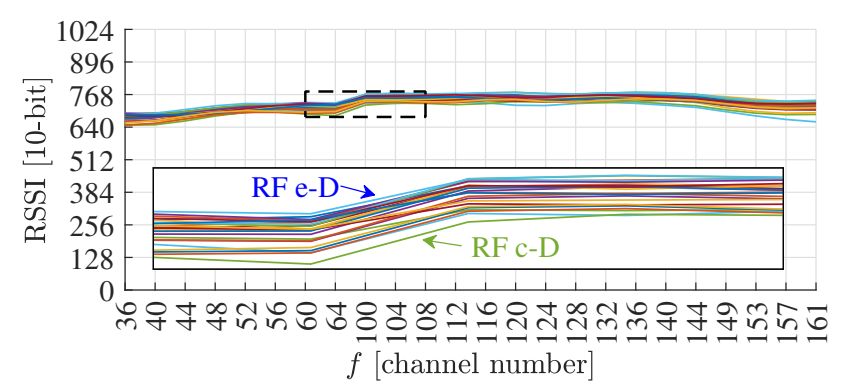

(a) RSSI vs. central frequency. Each curve represents an RF.

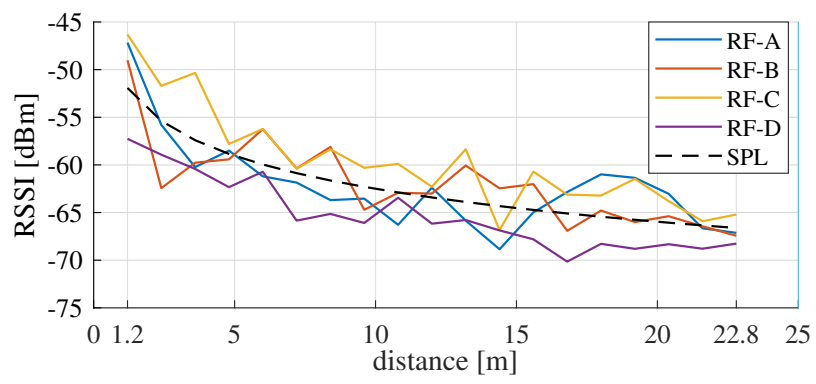

(b) RSSI vs. distance. The curve named SPL stands for the simplified path loss model with $\alpha=1.15$.

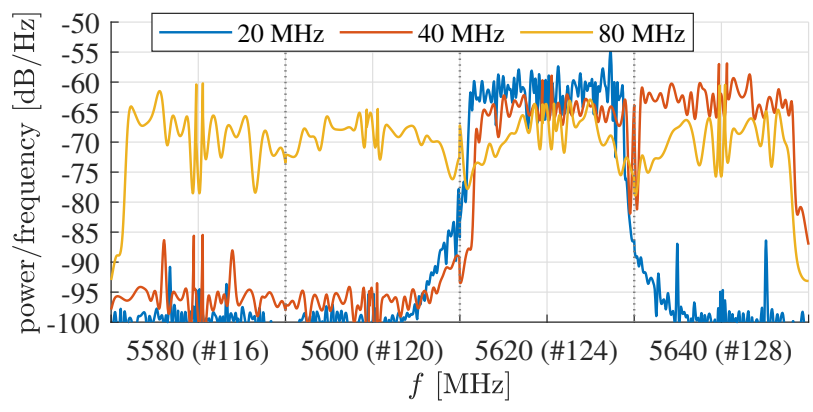

(c) Transmission bandwidth effect on power.

Figure 3: Validation metrics.

\subsubsection{Controlled testbed evaluation}

We now measure the activity of 3 overlapping BSSs' with static channel bonding capabilities under different traffic regimes. As shown in Figure 2, we separated the APs from their corresponding iperf servers by the same distance $d=4.8 \mathrm{~m}$. The WACA platform was placed equidistantly from the central AP and STA also at $d=4.8 \mathrm{~m}$.

We analyze two particular setups where none and all the BSSs' saturate. The load of every BSS is 20 and $150 \mathrm{Mbps}$ in the first and second setup, respectively. Table 2 collects the setups' details. Essentially, in the unsaturated scenario, all the BSSs' share the same primary channel (48) but are set with different bonding capabilities. The second setup assigns different primary channels and bandwidth capabilities to each BSS. Note that we changed the primary channels from the previous experiments due to AP hardware restrictions. Nonetheless, we also confirmed that the external interference at the new band of interest was negligible. 


\begin{tabular}{ccccccc}
\hline Regime & AP & 36 & 40 & 44 & 48 & Thr. [Mbps] \\
\hline \multirow{2}{*}{ Unsaturated } & AS & & & & $\mathbf{p}$ & 20 \\
$\ell=20$ Mbps & TP & & & & $\mathbf{p}$ & 20 \\
& LS & & & & $\mathbf{p}$ & 20 \\
Saturated & AS & & $\mathbf{p}$ & & & 100 \\
$\ell=150$ Mbps & TP & p & & & & 59 \\
& LS & & & p & 16 \\
\hline
\end{tabular}

Table 2: Setup of controlled experiments. Letter p indicates the primary channel whereas colors represent the allocated bandwidth to each AP.

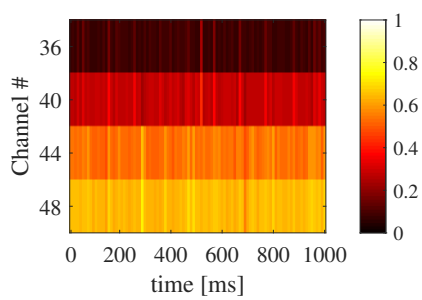

(a) Unsaturated.

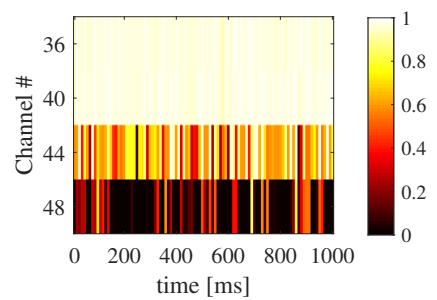

(b) Saturated.
Figure 4: Spectogram evolution of the controlled scenarios. Slots represent the 10 -ms mean occupancy.

WACA collected 1 second of consecutive RSSI samples during the execution of each setup to measure the spectrum occupancy. Figure 4 shows the occupancy evolution at each basic channel for the unsaturated and saturated setups. We confirm that the former setup does not reach full occupancy in any channel, whereas it is the contrary for the later. Indeed, we observe that the load $(\ell=150 \mathrm{Mbps})$ is high enough to make channels 36 and 40 occupied almost all the time (i.e., occupancy at the first $40-\mathrm{MHz}$ band is always close to 1) while not being able to successfully deliver all the load as indicated by the throughput values in Table 2. This has an important consequence for the LS AP. Essentially, since static channel bonding is applied, the LS AP does not benefit from having its primary channel (48) far from the rest of APs. Consequently, its throughput performance is drastically reduced given that AS and TP introduce inadmissible activity in the first $40 \mathrm{MHz}$ band.

\section{Measurement Campaigns}

Using WACA, we perform extensive measurement campaigns covering two continents, dense urban areas, and multiple hours of samples in places of interest such as university campuses, apartment buildings, shopping malls, hotels and the Futbol Club Barcelona (a.k.a Barça) stadium (Camp Nou), one of the largest sports stadiums in the world. The measurements were taken from February to August 2019 in Houston, TX, USA, and Barcelona, Spain. The shortest experiment took 20 minutes and the longest covered more than 1 week. The list of locations is shown in Table 3 .

\begin{tabular}{cccc}
\hline Id & Location & Type & Duration \\
\hline 1_RVA & Rice Village Apart., HOU & Apartment & 1 day \\
2_RNG & RNG lab at Rice, HOU & Campus & 1 day \\
3_TFA & Technology for All, HOU & Com. center & 1 day \\
4_FLO & Flo Paris, HOU & Cafe & 1 hour \\
5_VIL & Rice Village, HOU & Shopping mall & 20 min \\
\hline 6_FEL & La Sagrera, BCN & Apartment & 1 week \\
7_WNO & WN group office, BCN & Campus & 1 day \\
8_22@ & 22@ area, BCN & Office area & 1 day \\
9_GAL & Hotel Gallery, BCN & Hotel & 1 day \\
10_SAG & Sagrada Familia, BCN & Apartment & 4 days \\
11_FCB & Camp Nou, BCN & Stadium & 5 hours \\
\hline
\end{tabular}

Table 3: Measurement locations.

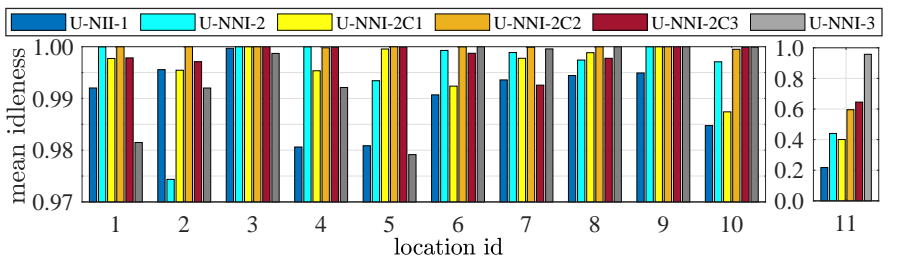

(a) Mean idleness per band.
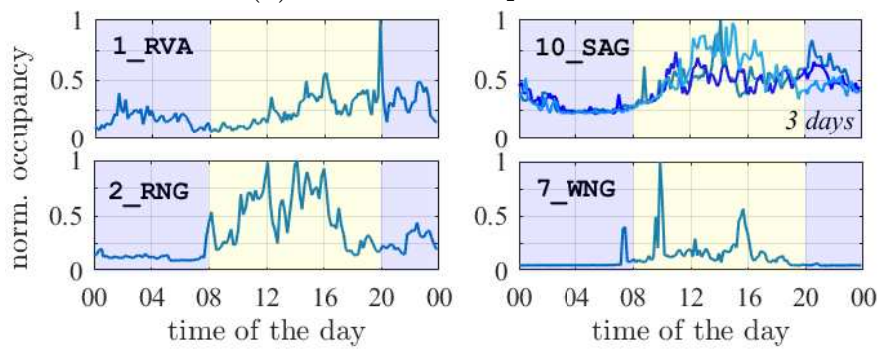

(b) Temporal evolution of the normalized occupation for apartments (upper) and universities (lower) highlighting day (yellow) and night (blue).

Figure 5: General spectrum occupancy trends.

Here, we overview the complete dataset: there are 153,033 iterations of 1 second accounting for 42 hours, 30 minutes, and 3 seconds of actual measurements in the $2420-\mathrm{MHz}$ channels of the $5 \mathrm{GHz}$ WiFi band. We first assess the entire activity record of each location at band $\mathcal{B}$, where $\mathcal{B}$ is a predefined set of contiguous channels. For instance, UNII-1 band is defined as $\mathcal{B}=\{1,2,3,4\}$, corresponding to basic channels 36, 40, 44, and 48 (see Figure 1a) ${ }^{6}$ Figure $5 a$ shows the normalized mean idleness of each band, i.e., the mean number of samples that were found idle in each channel of band $\mathcal{B}$. We observe that the spectrum is idle most of the time in all scenarios except the stadium, indicating that the $5 \mathrm{GHz}$ band is still profoundly underutilized even in densely populated areas.

Figure $5 \mathrm{~b}$ shows the daily temporal evolution of 4 exam-

${ }^{6}$ The rest of bands are sequentially composed of groups of four consecutive $20-\mathrm{MHz}$ channels. So, the next band is U-NNI-2 with channels $\{5,6,7,8\}$ (basic channels 52, 56, 60, and 64), and the last one is UNNI-3 with channels $\{21,22,23,24\}$ (basic channels $149,153,157,161$ ). 
ple locations (2 apartments in the upper subplots, and 2 university campuses in the lower subplots). For the sake of representation, we plot the normalized occupancy of the whole band averaged in periods of 10 minutes. Concretely, we normalize with respect to the highest 10-minutes average occupancy encountered in each location. We clearly observe higher activity at working hours in the campus locations and a much less variable pattern in the apartment locations. In any case, from the low spectrum utilization observed in Figure 5 a significant opportunities for channel bonding can be expected.

Figure 6a shows a photograph of the WACA setup in the Futbol Club Barcelona's stadium. We deployed WACA in the press box of the stadium during a football game with over 98,000 spectators present. Measurements were taken on August 4, 2019, from 17:24 to 22:30 accounting for a total duration of 5 hours and 6 minutes. On that date, the Joan Gamper trophy was held, which pitted the local club (Futbol Club Barcelona) against the visiting club (Arsenal Football Club). Free Wi-Fi was provided to the audience through thousands of APs primarily installed beneath the seats. We also obtained data from the stadium's network management team which indicated that up to $12,000 \mathrm{Wi}-\mathrm{Fi}$ clients were simultaneously connected. Downlink and uplink traffic is depicted in Figure 6b and the spectogram in Figure 6c Each slot in the spectogram represents the occupancy of each channel averaged in 1-second periods. We observe that most channels were highly occupied during the measurements. Moreover, we can observe the users' behavior induced nonstationarity of the traces as the match progressed. Namely, while activity is always high, there is a notable reduction during play (first and second half) compared to activity before, between halfs, and right after the game time. We also observe that the majority of the channels are crowded most of the time with periods reaching mean band occupancy values rising up to $99 \%$. In fact, $22 \%$ of the periods are above $80 \%$ occupancy.

\section{$5 \quad$ Evaluation of bonding gains}

In this section, we study the performance of channel bonding against the band occupancy. Notice that, due to the disparate nature of the stadium scenario (recall the contrasting mean idleness in Figure 5a, we focus our study on the non-stadium measurement campaigns to provide insights on a more homogeneous dataset. The analysis of the stadium campaign will be covered in future work.

\subsection{Evaluation Methodology}

\subsubsection{Overview}

Our objective is to study the throughput performance that a fully backlogged channel-bonding BSS, $w$, would obtain if it

\footnotetext{
${ }^{7}$ Aggregated downlink and uplink traffic was provided by Futbol Club Barcelona's IT management.
}

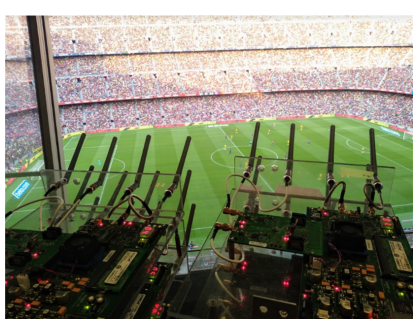

(a) WACA deployment.

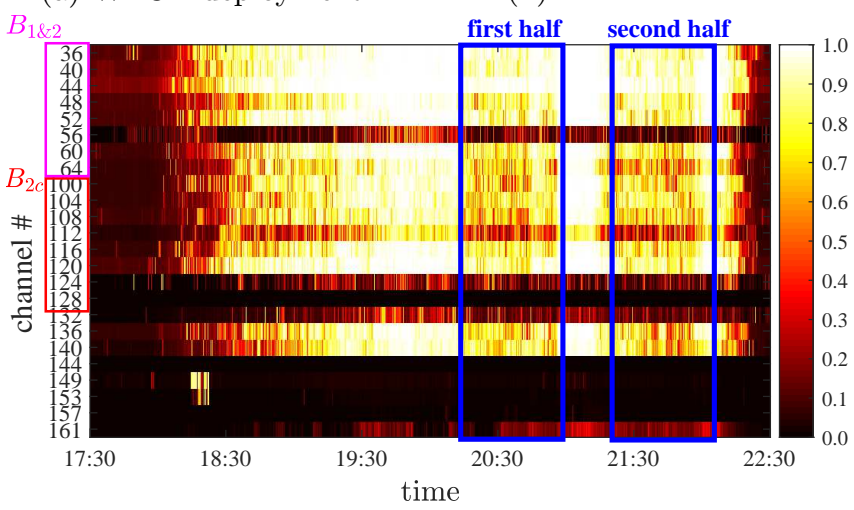

(c) Spectrum occupancy evolution.

Figure 6: Wi-Fi activity at the Camp Nou stadium: a) WACA deployment in the press box, b) downlink and uplink Wi-Fi traffic evolution (source: stadium's IT management), and c) spectrum occupancy evolution.

encountered the channels recorded in the measurement campaigns described above. An schematic of the system model is illustrated in Figure 7. BSS $w$ consists of an access point (AP) and a one or multiple stations (STAs) sufficiently close to assume that they perceive the same RSSI measurements.

The throughput is a function of a number of factors such as which primary channel the bonding BSS selects, which channel bonding policy it employs, and the spectrum occupancy perceived while operating. So, here we describe the tracedriven framework we use. The RSSI measurements captured by WACA at scenario $s$ are represented by a 2-dimensional

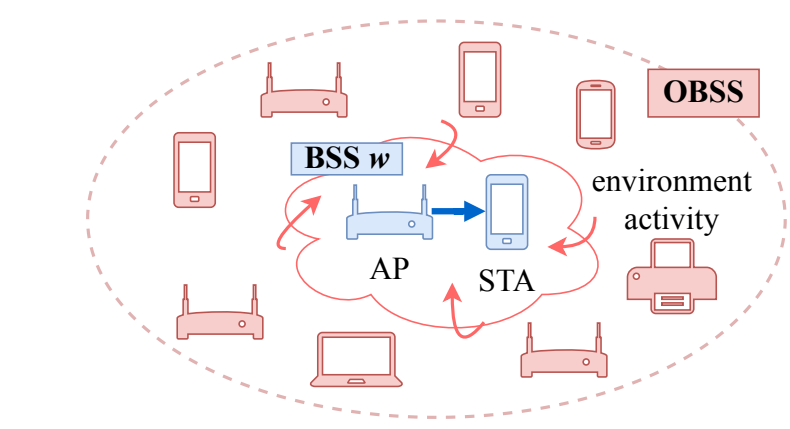

Figure 7: Diagram of the system model. The fully backlogged channel-bonding BSS, $w$, operates under instances of the same environment - or overlapping BSS (OBSS) - that WACA perceived at the measurement campaigns. 
matrix $Y^{s}$ of size $\left(N_{\mathrm{it}}^{s} \times n_{\mathrm{s}}\right) \times|\mathcal{R}|$, where $N_{\mathrm{it}}^{s}$ is the number of iterations of scenario $s$, and any element $y_{t, c}^{s}$ represents the power value at temporal sample $t$ in basic channel $c$. From $Y^{s}$, we generate a binary matrix $X^{s}$ of same size through an occupancy indicator function, where any element $x_{t, c}^{s}$ represents whether channel $c$ was occupied at temporal sample $t$ (1) or not (0). Formally, $x_{t, c}^{s}=\left(y_{t, c}^{s}>\right.$ CCA : 1,0$), \forall t, c$, where the clear channel assessment (CCA) is set to -83.5 $\mathrm{dBm}$ (or 150 10-bit RSSI units), corresponding to the common CCA threshold $-82 \mathrm{dBm}$ plus a safety margin of -1.5 $\mathrm{dBm}$. While IEEE 802.11ac/11ax introduce different CCA levels for the primary and secondary channels, in this work, we consider a more restrictive approach by assuming the same threshold in order to fairly compare different channel bonding policies. The mean occupancy at a certain band $\mathcal{B}$ in scenario $s$ is simply defined as

$$
\bar{o}_{\mathcal{B}}^{s}=\frac{\sum_{t} \sum_{c \in \mathcal{B}} x_{t, c}^{s}}{N_{\mathrm{it}}^{s} n_{\mathrm{s}}|\mathcal{B}|} .
$$

To provide meaningful experiments, we separately consider two $160-\mathrm{MHz}$ bands composed of 8 basic channels: the U-NII-1\&2 and part of the U-NII-2c sub-bands, $\mathcal{B}_{1 \& 2}=\{1,2,3, \ldots, 8\}$ and $\mathcal{B}_{2 \mathrm{c}}=\{9,10,11, \ldots, 16\}$, respectively. These sub-bands cover from channel 36 to 64 and from channel 100 to 128, respectively (see Figure 1a). Notice that these are the only sub-bands that allow to perform 160-MHz transmissions in the IEEE 802.11ac/ax channelization. Moreover, we focus on epochs (or periods) of duration $T_{\text {per }}=100 \mathrm{~ms}$ for which the mean occupancy at such subbands is at least $5 \%$, i.e., $\bar{o}_{\mathcal{B}} \geq 0.05$, where $\mathcal{B} \in\left\{\mathcal{B}_{1 \& 2}, \mathcal{B}_{2 \mathrm{c}}\right\}$.

\subsubsection{State Machine}

We develop a discrete state machine that characterizes how the channel-bonding BSS responds to each power sample (or temporal sample) $t$ according to the current state $S(t)$, and channel-bonding policy $\pi$, following the 802.11 standard. The set of possible states is $\mathcal{S}=\{$ Busy, DIFS, BO,TX/RX\}. State Busy indicates that the primary channel is busy, $D I F S$ represents the period before initiating the backoff process, the backoff counter is decreased during $B O$ state, and $T X / R X$ represents the actual frame transmission-reception (including the control frames RTS, CTS, and ACK, the DATA frame, and the SIFS periods in between). We represent the channel-bonding BSS $w$ as an AP and one or multiple clients that would perceive exactly the same spectrum activity as WACA captured in the measurement campaigns and must contend accordingly.

The set of basic channels selected for transmitting a frame depends both on the spectrum occupancy and on the selected channel-bonding policy $\pi$. Figure 8 illustrates an example of the transitions between states. Empty slots have a duration $T_{\text {slot }}=10 \mu \mathrm{s}$ and we consider $T_{\text {slot }}=T_{\mathrm{s}}=10 \mu \mathrm{s}$ rather than 9 us (802.11's default value) to align the duration of an idle backoff slot with the sample duration. Hence, whenever the channel-bonding BSS is in the backoff process at state

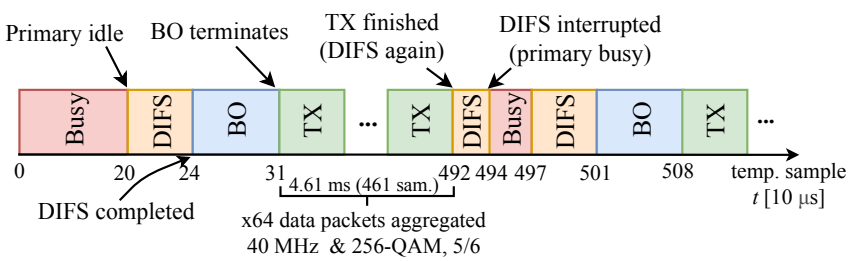

Figure 8: Example of the transitions between states.

$B O$, every idle sample at the primary channel $p$ results in a backoff counter decrease of one empty slot. We use Wi-Fi parameters according to IEEE 802.11ax 3 and assume 256QAM modulation coding scheme regardless of the transmission bandwidth. The setup parameters are listed in Table 4. After running the state-machine through all the temporal samples in the epoch, we compute the throughput $\Gamma$ as the number of data packets sent divided by the duration of the epoch $T_{\text {per }}$.

\begin{tabular}{ccc}
\hline Param. & Description & Value \\
\hline CCA & CCA threshold & $-83.5 \mathrm{dBm}$ \\
MCS & MCS index & $9(256-\mathrm{QAM} 5 / 6)$ \\
$b$ & basic channel bandwidth & $20 \mathrm{MHz}$ \\
$L_{\mathrm{d}}$ & Length of a data packet & $12000 \mathrm{bit}$ \\
$\mathcal{B}$ & Allocated set of basic channels & $\mathcal{B} \subseteq \mathcal{R}$ \\
$p$ & Primary channel & $p \in \mathcal{B}$ \\
$N_{\mathrm{a}}$ & Max. no. of agg. packets per frame & 64 \\
$T_{\mathrm{e}}$ & Duration of an empty slot & $10 \mu \mathrm{s}$ \\
$T_{\mathrm{SIFS}}$ & SIFS duration & $20 \mu \mathrm{s}$ \\
$T_{\mathrm{DIFS}}$ & DIFS duration & $30 \mu \mathrm{s}$ \\
$T_{\mathrm{PIFS}}$ & PIFS duration & $30 \mu \mathrm{s}$ \\
$T_{\mathrm{RTS}}$ & RTS duration & $50 \mu \mathrm{s}$ \\
$T_{\mathrm{CTS}}$ & CTS duration & $40 \mu \mathrm{s}$ \\
$T_{\mathrm{BACK}}$ & Block ACK duration & $50 \mu \mathrm{s}$ \\
TXOP & Max. duration of a TXOP & $5 \mathrm{~ms}$ \\
$\mathrm{CW}$ & 16 \\
$m$ & Min. contention window & 5 \\
\hline
\end{tabular}

Table 4: Trace-driven setup.

\subsubsection{Channel Bonding Policies and Response}

A channel-bonding policy $\pi$ selects the set of basic channels to aggregate at the end of the backoff provided that the primary channel is available. Namely, contiguous channel bonding can select a set of channels both above and below the primary channel, provided they are consecutive. In contrast, non-contiguous channel bonding can combine all available channels at the time the primary channel becomes available. We compare both policies in $\$ 5.2$.

How will other BSS's respond to the channel bonding BSS? We consider that they will defer their transmissions. Namely, the channel bonding BSS needs the channels to be available only when its countdown timer expires. If the bonding BSS does transmit but the trace indicates that a channel would 
have been occupied at some point during the transmission, we consider that such other BSS's will sense the bonding BSS and defer.

\subsection{Contiguous vs. Non-Contiguous}

For each transmission, non-contiguous channel bonding can utilize additional channels as compared to contiguous, by "skipping over" the busy channels to find the next unused one. Here, we explore the gains of this flexibility as well as (in rare cases) the losses by comparing the throughput of contiguous and non-contiguous channel bonding in three load regimes: low $\left(\bar{o}_{\mathcal{B}} \leq 0.1\right)$, medium $\left(0.1<\bar{o}_{\mathcal{B}} \leq 0.2\right)$ and high $\left(\bar{o}_{\mathcal{B}}>0.2\right)$, respectively.

Figure 9 shows the the throughput ratio of contiguous to non-contiguous channel bonding $\Gamma_{\mathrm{CO}}(p) / \Gamma_{\mathrm{NC}}(p)$, where $\Gamma_{\pi}(p)$ is the throughput achieved by policy $\pi$ when selecting primary $p$ in a given period. We plot the ratio for all possible primaries in $\mathcal{B}_{1 \& 2}$ and $\mathcal{B}_{2 \mathrm{c}}$.

The data reveals two remarkable phenomenon. First, contiguous outperforms non-contiguous in $2.5 \%$ of the cases (albeit with a modest throughput difference of $1.9 \%$ ). But since non-contiguous is more flexible, how can it ever do worse? The answer is that the two policies result in different instants for transmission attempts. The contiguous policy occasionally (and quite randomly since the traces are the same) ends up with more favorable attempt instants. Nonetheless, in most cases, non-contiguous obtains higher throughput. For example, in many periods, at least one $20-\mathrm{MHz}$ channel is idle during the whole period, which will always yield a gain for non-contiguous, but only sometimes yields a gain for contiguous bonding. In some cases, the difference can be quite high (e.g., a ratio of approximately 0.2 observed in low load). The origins of such extreme cases are the selection of the primary channel. Second, the bar chart inside Figure 9 reveals that both contiguous and non-contiguous channel bonding perform quite close on average for all occupancy regimes (low, medium, and high), and especially for the latter, as high load results in far fewer bonding opportunities overall.

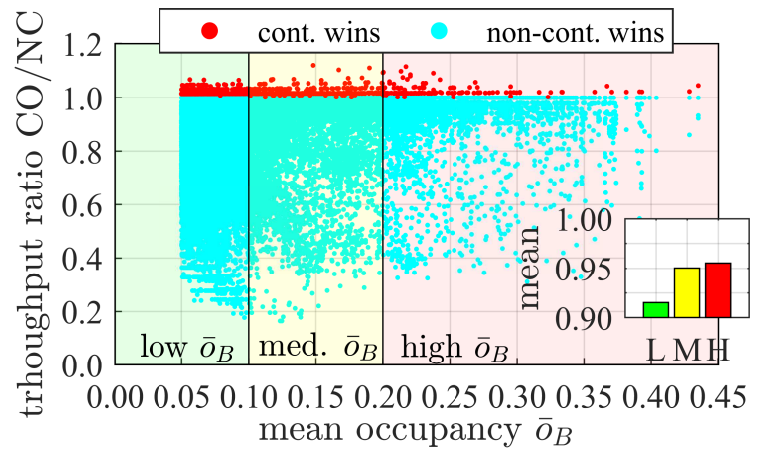

Figure 9: Throughput ratio of contiguous vs. non-contiguous channel bonding. The bar chart inset depicts the mean aggregated ratio for low (L), medium (M), and high $(\mathrm{H})$ occupancy regimes.
Finding: Non-contiguous almost always outperforms contiguous channel bonding and their throughput differences are occasionally over a factor of 5 . Nonetheless, their average throughputs are quite similar, which may ultimately favor contiguous channel bonding, since it is simpler to implement.

\section{Conclusion}

In this paper, we introduce WACA, an all-channel Wi-Fi spectrum analyzer for simultaneous measurement of all 24 $20 \mathrm{MHz}$ channels that allow channel bonding at $5 \mathrm{GHz}$. We present extensive measurement campaigns covering two continents, diverse areas, and many hours of signal strength samples. The gathered dataset is a unique asset to find insights otherwise not possible to study. We leave the analysis of key factors like spectral correlation or bandwidth prevention as future work.

\section{Acknowledgements}

We appreciate the generosity of Futbol Club Barcelona for letting us take measurements in the Camp Nou stadium. The authors would also like to thank Chia-Yi Yeh, Albert Bel, lvaro Lpez, and Marc Carrascosa for their contribution in taking the measurements. The work from S. BarrachinaMuoz and B. Bellalta has been partially supported by CISCO (CG\#890107-SVCF), Maria de Maeztu Units of Excellence Programme (MDM-2015-0502), WINDMAL PGC2018099959-B-I00 (MCIU/AEI/FEDER,UE), and SGR-20171188. The research of E. Knightly was supported by Cisco, Intel, and by NSF grants CNS-1955075 and CNS-1824529.

\section{References}

[1] IEEE 802.11n enhancements for Higher Throughput, 2009.

[2] IEEE 802.11ac Very High Throughput., 2013.

[3] IEEE P802.11ax/D4.0 High Efficiency WLAN, 2019.

[4] IEEE 802.11be Extremely High Throughput, 802.1118/1231r6, 2019.

[5] Michael Rademacher, Karl Jonas, and Mathias Kretschmer. Quantifying the spectrum occupancy in an outdoor $5 \mathrm{GHz}$ WiFi network with directional antennas. In 2018 IEEE Wireless Communications and Networking Conference (WCNC), pages 1-6. IEEE, 2018.

[6] Tanim Taher, Ryan Attard, Ali Riaz, Dennis Roberson, Jesse Taylor, Kenneth Zdunek, Juhani Hallio, Reijo Ekman, Jarkko Paavola, Jaakko Suutala, et al. Global spectrum observatory network setup and initial findings. 
In 2014 9th International Conference on Cognitive Radio Oriented Wireless Networks and Communications (CROWNCOM), pages 79-88. IEEE, 2014.

[7] Sriram Subramaniam, Hector Reyes, and Naima Kaabouch. Spectrum occupancy measurement: An autocorrelation based scanning technique using USRP. In 2015 IEEE 16th Annual Wireless and Microwave Technology Conference (WAMICON), pages 1-5. IEEE, 2015.

[8] Matthias Wellens, Janne RiihijäRvi, and Petri MäHöNen. Empirical time and frequency domain models of spectrum use. Physical Communication, 2(12):10-32, 2009.

[9] Vinod Kone, Lei Yang, Xue Yang, Ben Y Zhao, and Haitao Zheng. The effectiveness of opportunistic spectrum access: A measurement study. IEEE/ACM Transactions on Networking, 20(6):2005-2016, 2012.

[10] Simon W Day and Adrian Wagstaff. Activity in the WiFi bands - An Objective Approach to Estimation. In ARMMS Conference. Mass, 2014.

[11] Fatima Salahdine and Hassan El Ghazi. A real time spectrum scanning technique based on compressive sensing for cognitive radio networks. In 2017 IEEE 8th Annual Ubiquitous Computing, Electronics and Mobile Communication Conference (UEMCON), pages 506511. IEEE, 2017.

[12] Sanjit Biswas, John Bicket, Edmund Wong, Raluca Musaloiu-e, Apurv Bhartia, and Dan Aguayo. Largescale measurements of wireless network behavior. In Proceedings of the 2015 ACM Conference on Special Interest Group on Data Communication, pages 153-165, 2015.

[13] M. Lpez-Bentez and F. Casadevall. Modeling and simulation of time-correlation properties of spectrum use in cognitive radio. In 2011 6th International ICST Conference on Cognitive Radio Oriented Wireless Networks and Communications (CROWNCOM), pages 326-330, 2011.

[14] Vinod Kone, Lei Yang, Xue Yang, Ben Y Zhao, and Haitao Zheng. On the feasibility of effective opportunistic spectrum access. In Proceedings of the 10th $A C M$ SIGCOMM conference on Internet measurement, pages 151-164, 2010.

[15] Marko Höyhtyä, Aarne Mämmelä, Marina Eskola, Marja Matinmikko, Juha Kalliovaara, Jaakko Ojaniemi, Jaakko Suutala, Reijo Ekman, Roger Bacchus, and Dennis Roberson. Spectrum occupancy measurements: A survey and use of interference maps. IEEE Communications Surveys \& Tutorials, 18(4):2386-2414, 2016.
[16] Salim A Hanna and John Sydor. Distributed sensing of spectrum occupancy and interference in outdoor 2.4 $\mathrm{GHz}$ Wi-Fi networks. In 2012 IEEE Global Communications Conference (GLOBECOM), pages 1453-1459. IEEE, 2012.

[17] Salim A Hanna. A 3-state hypothesis test model for cognitive radio systems. In 2014 IEEE International Symposium on Dynamic Spectrum Access Networks (DYS$P A N)$, pages 291-302. IEEE, 2014.

[18] Sixing Yin, Dawei Chen, Qian Zhang, Mingyan Liu, and Shufang Li. Mining spectrum usage data: a large-scale spectrum measurement study. IEEE Transactions on Mobile Computing, 11(6):1033-1046, 2012.

[19] Lara Deek, Eduard Garcia-Villegas, Elizabeth Belding, Sung-Ju Lee, and Kevin Almeroth. The impact of channel bonding on 802.11n network management. In Proceedings of the Seventh COnference on emerging Networking EXperiments and Technologies, pages 1-12, 2011.

[20] Lara Deek, Eduard Garcia-Villegas, Elizabeth Belding, Sung-Ju Lee, and Kevin Almeroth. Intelligent channel bonding in $802.11 \mathrm{n}$ WLANs. IEEE Transactions on Mobile Computing, 13(6):1242-1255, 2014.

[21] Mustafa Y Arslan, Konstantinos Pelechrinis, Ioannis Broustis, Srikanth V Krishnamurthy, Sateesh Addepalli, and Konstantina Papagiannaki. Auto-configuration of 802.11n WLANs. In Proceedings of the 6th International Conference, pages 1-12, 2010.

[22] Mustafa Y Arslan, Konstantinos Pelechrinis, Ioannis Broustis, Shailendra Singh, Srikanth V Krishnamurthy, Sateesh Addepalli, and Konstantina Papagiannaki. ACORN: An auto-configuration framework for 802.11n WLANs. IEEE/ACM Transactions on Networking (TON), 21(3):896-909, 2013.

[23] Minyoung Park. IEEE 802.11ac: Dynamic bandwidth channel access. In Communications (ICC), 2011 IEEE International Conference on, pages 1-5. IEEE, 2011.

[24] Michelle X Gong, Brian Hart, Liangfu Xia, and Roy Want. Channel bounding and mac protection mechanisms for 802.11ac. In 2011 IEEE Global Telecommunications Conference-GLOBECOM 2011, pages 1-5. IEEE, 2011.

[25] Boris Bellalta, Alessandro Checco, Alessandro Zocca, and Jaume Barcelo. On the interactions between multiple overlapping wlans using channel bonding. IEEE Transactions on Vehicular Technology, 65(2):796-812, 2015.

[26] Mohand Yazid and Adlen Ksentini. Stochastic modeling of the static and dynamic multichannel access methods enabling 40/80/160 MHz channel bonding in the VHT 
WLANs. IEEE Communications Letters, 23(8):14371440, 2019.

[27] Mun-Suk Kim, Tanguy Ropitault, Sukyoung Lee, and Nada Golmie. A throughput study for channel bonding in ieee 802.11ac networks. IEEE Communications Letters, 21(12):2682-2685, 2017.

[28] Sergio Barrachina-Muñoz, Francesc Wilhelmi, and Boris Bellalta. To overlap or not to overlap: Enabling channel bonding in high-density WLANs. Computer Networks, 152:40-53, 2019.

[29] Sergio Barrachina-Muoz, Francesc Wilhelmi, and Boris Bellalta. Dynamic channel bonding in spatially distributed high-density WLANs. IEEE Transactions on Mobile Computing, 19(4):821-835, 2020.

[30] Giorgio Buttazzo, Carmelo Di Franco, and Mauro Marinoni. Design and analysis of target-sensitive realtime systems. Software: Practice and Experience, 46(9):1181-1200, 2016.

[31] Leili Farzinvash and Mehdi Kargahi. A scheduling algorithm for execution-instant sensitive real-time systems. In 2009 15th IEEE International Conference on Embedded and Real-Time Computing Systems and Applications, pages 511-518. IEEE, 2009.

[32] Narendra Anand, Ehsan Aryafar, and Edward W Knightly. WARPlab: a flexible framework for rapid physical layer design. In Proceedings of the 2010 ACM workshop on Wireless of the students, by the students, for the students, pages 53-56. ACM, 2010.

[33] Rice University Wireless Open Access Research Platform. http://warpproject.org, last accessed January 30,2020 .

[34] Seowoo Jang, Kang G Shin, and Saewoong Bahk. Post$\mathrm{CCA}$ and reinforcement learning based bandwidth adaptation in 802.11ac networks. IEEE Transactions on Mobile Computing, 17(2):419-432, 2018. 